\title{
DREAM THEATER: \\ KOMPONIEREN IM VIRTUOSENKOLLEKTIV
}

\section{Michael Custodis}

Virtuosität entfaltet in solchen Momenten eine besondere Faszination, wenn dank ausgefeilter technischer Fähigkeiten mit großer Sorgfalt, Routine und Gelassenheit komplizierte Zusammenhänge so transparent werden, dass die Komplexität dieser Vorgänge sich wie von selbst zu erklären scheint. Und dennoch verbleibt im Feld der Musik ein fast mystischer, unerklärlicher Rest von Unverständnis auf Seiten des Publikums. Auch präzise spieltechnische Analysen können nicht in Gänze erfassen, weshalb gerade die Virtuosität von Musikern auf die Emotionalität ihrer Zuschauer besonders einwirkt. Die Musiker wiederum betrachten sich selbst selten in der abstrakten Pose eines Virtuosen. Sie arbeiten sich selbstkritisch an den Grenzen ihrer musikalischen Ausdrucksmittel ab, die sie von noch größerer musikalischer Perfektion trennen, wenn dies ihr Ziel ist. Ihre Spieltechnik ist daher nur Mittel zum Zweck, nicht aber Selbstzweck. Um aus der Sicht des Publikums auf Virtuosen und aus der Selbstwahrnehmung von Virtuosen dieses Wechselverhältnis von technischer Versiertheit und musikalischer Ausdrucksfähigkeit besser zu verstehen, scheinen einige historische Bemerkungen vorab hilfreich.

Soweit historische Quellen zurückreichen, wurden virtuose Musiker vor allem für ihr Talent bewundert, mit außergewöhnlichen Fertigkeiten die Gefühle ihrer Zuhörer zu rühren, angefangen bei Gott Apollo und Orpheus, der sogar Tiere und unbelebte Materie zu erweichen wusste. Klischees von Virtuosität als göttlicher Gabe, als Teufelspakt oder artistisch-sportlicher Leistungsschau bestimmen seither die Debatten, deutlich intensiviert mit dem Aufkommen italienischer und deutscher Musikästhetiken um das Jahr 1700 (Mattheson 1722: 114; Stößel 1749: 418). Johann Kuhnau widmete diesem Thema einen eigenen Roman, dessen Titel Der musicalische Quacksalber bereits die Tendenz andeutet, Virtuosität bei mangelnder Charakterstärke als Domäne von Scharlatanen und Betrügern zu klassifizieren. Nicht zufällig ist es daher die Sphäre des Religiösen, die nach vielen Kapiteln den 
Protagonisten Caraffa auf den rechten Weg zurückführt, als ihm ein Priester zur Vergebung seiner Sünden einen Bußkatalog von 64 Paragraphen mit dem Titel Der wahre Virtuose und glückselige Mensch überreicht. Abgesehen von nationalistischen Vorurteilen gegen italienische Oberflächlichkeit ist der achte Paragraph besonders aufschlussreich, der die wahre Virtuosität eines Künstlers als Einheit theoretischer Kenntnisse, kompositorischer Erfahrung und instrumentaler Praxis definiert (Kuhnau 1700: 500).

Die Personalunion von Komponisten und Interpreten als Virtuosen verlor sich im Verlauf des 18. Jahrhunderts, bis im Begriff der »Werktreue « die Hierarchie von kompositorischem Genie und ausführenden Interpreten so dominant wurde, dass Virtuosität insgesamt in Verruf geriet (Dahlhaus/ Eggebrecht 1967: 1047f.; Reimer 1972). Leopold Mozarts Lehrbücher unterschieden entsprechend zwischen einer echten und falschen Form von Virtuosität als Differenz von geschmackvoller Eleganz und der Vorführung rein mechanischer Brillanz (Mozart 1770: 262f.). Im anschließenden 19. Jahrhundert konnten es reisende Virtuosen im expandieren europäischen Musikmarkt zu Ruhm und großem Reichtum bringen, wobei vor allem die Geschmäcker von Publikum, Verlegern und Konzertveranstaltern die Auswahl und Gestaltung erfolgversprechenden Repertoires beeinflussten. So sehr Kritik und Publikum Interpreten wie Niccolo Paganini und Franz Liszt frenetisch feierten, so sehr misstrauten sie zugleich deren kompositorischem Können, was die Lisztforschung bis heute beschäftigt. Auch Alban Berg verbat sich im Programm für Arnold Schönbergs Wiener Verein für musikalische Privataufführungen (1918-21) mit einem eigenen Paragraphen explizit jedes Zurschaustellen von Virtuosität (Berg 1984: 5; Jütte 2009; Riethmüller 2001).

Vergleichbar der Zwiespältigkeit, mit der man Virtuosität in der klassischen Musik bis heute beäugt und zugleich insbesondere auf der Opernbühne feiert, wird sie auch im Bereich der Rockmusik vom Publikum in Form exzentrischer Soli erwartet und ist zugleich der Kritik ausgesetzt. Insbesondere dort, in der Rockmusik, kommt die etymologische Ableitung des Virtuosen vom lateinischen Wort »vir« für »Mann« als genderspezifisches Element zum Tragen (Bork 2010: 510f.). Abgesehen von Sängerinnen wird Rockmusik in den allermeisten Fällen bis heute von männlichen Instrumentalisten dominiert, ohne dass bislang eine soziologisch befriedigende Erklärung für dieses Phänomen gefunden worden wäre.

Erwartungen, Vorurteile und Bewunderung - allen diesen Perspektiven gemeinsam ist der Blick auf Virtuosen. Als Ausnahmekünstler bewerten wir zwar ihre technischen Fähigkeiten nach mehr oder weniger sachlichen Kriterien. Gleichzeitig aber steht immer auch die ethische Angemessenheit zur Disposition, eine solche technische Überlegenheit stolz oder bescheiden zur 
Schau zu stellen. Wie aber denken solche Künstler selbst über Virtuosität und wie setzen sie diese gezielt als dramatisches Mittel ein? Denn Virtuosität ist weniger eine Kategorie, die der Selbstwahrnehmung von Künstlern entspricht, sondern vielmehr - in der umgekehrten Blickrichtung - eine Rezeptionsweise bei der Betrachtung solcher Ausnahmemusiker. Erläutern lässt diese These am Beispiel der Band Dream Theater in einem methodischen Zweischritt: Da Virtuosität untrennbar an die Live-Präsenz von Musikern gebunden ist, um einer staunenden und begeisterten Zuhörerschaft die Finessen einer Komposition oder Improvisation buchstäblich vor Augen zu führen, thematisiert der erste Punkt das spontane Interpretieren arrangierter und damit konstruierter Komplexität. Im Sinne einer Steigerung der Stilmittel nimmt der zweite Punkt das Komponieren in Dream Theaters Virtuosenkollektiv in den Blick. Erst hier, in der Balance hohen technischen Anspruchs (einschließlich aller Bereiche von Sound und Bühnenshow) und alternativer Stilmittel (z.B. ruhiger melodischer Passagen) ist zu studieren, wie Kontraste die Wirkung eingestreuter virtuoser Passagen steigern.

\section{Linearität und Komplexität}

Jenseits musikalischer Fähigkeiten beginnt technische Perfektion bei physiologischen Gegebenheiten, da Muskeln speziell entwickelt, Bewegungsabläufe permanent trainiert und kleineste Details so verinnerlicht werden müssen, bis aus ihrer musikalischen Verselbstständigung ein eigener Stil entstehen kann. Diesen Aspekt ständigen routinierten Trainings teilen Musiker mit Sportlern. Wenn zu außergewöhnlichem Fleiß noch außergewöhnliches Talent hinzukommt, erreichen solche Musiker ein grundlegendes Niveau, das für andere bereits virtuos und kaum erreichbar wirkt, während es für sie noch keine Herausforderung beinhaltet. Die Mitglieder der US-amerikanischen Progressive Metal-Band Dream Theater werden im Bereich der Rockmusik hierfür gerne als Vorbilder angeführt, ohne dass die Geschichte der 1985 von Schlagzeuer Mike Portnoy, Gitarrist John Petrucci und Bassist John Myung gegründeten Gruppe an dieser Stelle mit wegen Worten zusammenzufassen wäre. ${ }^{1}$ Festzustellen ist, dass bis zur heutigen Konstellation - 1991 kam Sänger James LaBrie hinzu, acht Jahre später Keyboarder Jordan Rudess sowie 2011 Schlagzeuger Mike Mangini - jede Umbesetzung die Musikalität und technische Finesse der Gruppe weiter steigerte, vor allem in der

1 Vergleiche zur Bandbiografie zahlreiche Fanseiten im Internet, insbesondere die von der Band und ihrem ehemaligen Mitglied Mike Portnoy selbst gepflegten Websites. 
Selbsteinschätzung ihres kreativen Potenzials. Die oft bemühten Vergleiche von Dream Theaters technischer Versiertheit zu Kollegen aus dem Bereich der klassischen Musik sind durchaus zutreffend, da alle nicht nur ihre Instrumente studierten - die meisten am Berklee College of Music, wo Magini bis zu seinem Einstieg bei Dream Theater auch als Professor unterrichtete, während Rudess im Solistenprogramm der New Yorker Juilliard School eingeschrieben war (Custodis 2016). Vielmehr pflegen alle bis heute auch eine entsprechend hohe individuelle Übungsroutine und kollektive Probendisziplin.

Wenn hinsichtlich der Bewertungsmaßstäbe festgestellt wurde, dass Parts auf durchschnittlichere Musiker bereits virtuos wirken, die für technisch extrem versierte Musiker noch keine Herausforderung darstellen, gilt dies insbesondere für das Zusammenspiel von Virtuosen. Wenn (wie im Fall von Dream Theater) die Interpreten ihre eigenen Stücke komponieren, lohnt ein Blick auf jene Passagen, die nicht als explizite Solostellen die Fertigkeit einzelner Musiker besonders hervorheben, sondern umgekehrt ihr Zusammenspiel betonen. Ein gutes Beispiel ist der Beginn des zwanzigminütigen Stücks »In The Presence Of Enemies «, das in zwei Teilen Dream Theaters 2007 erschienenes neuntes Studioalbum Systematic Chaos ${ }^{2}$ eröffnet und beschließt. Zu diesem Album wurde ein Songbook vorgelegt, in dem entlang der Noten verfolgt werden kann, wie durch Schichtungen und Verdichtungen die rhythmische Komplexität eines Songs langsam gesteigert wird und wie sie dank kurzer, gut zu identifizierender Motive dabei transparent bleibt. Wenn eine Schicht etabliert ist, wird sie von einer weiteren überlagert, die zunächst wie ein Solo wirkt und doch nur eine nächste Ebene bildet.

Richten wir für einen Moment, quasi mit einer musikanalytischen Lupe, den Blick auf den Beginn des Stücks: Die ersten vier Takte umspielen unisono mit Terzschritten einen abwärts geführten d-Moll-Akkord, bei dem der Tonraum einer Quinte kaum verlassen wird. Mit $D$ als Orgelpunkt übernimmt anschließend die Gitarre die Stimmführung und etabliert eine zweitaktige Einheit, bei der $D$ als repetierter Referenzton fungiert:

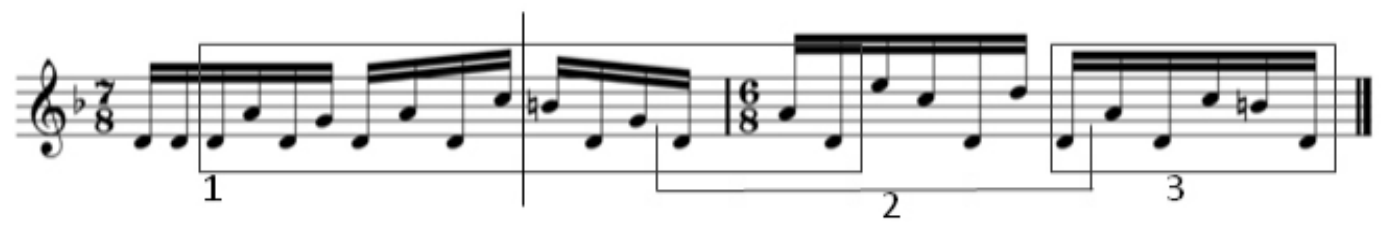

Abbildung 1: Dream Theater - »In The Presence Of Enemies«, Takt 5-6

2 Dream Theater (2007). Systematic Chaos. Roadrunner Records RR 7992-8. 
Auch wenn das Notenbild diese zweitaktige Einheit als Kombination eines 7/8- mit einem 6/8-Takt ausweist, überspielen die Gitarrenfiguren diesen Taktwechsel. Vergleicht man diese Stelle im Höreindruck, erklärt sich dieser Effekt als geschicktes Spiel mit musikalischen Erwartungen und konventionellen Hörweisen: Mit der dritten Wiederholung des $D$ beginnt eine charakteristische Pendelbewegung, die zunächst von den Intervallpaaren $D-A, D-G$ und wieder $D-A$ bis auf die Septime $D-C$ springt (Markierung 1$)$. Wie an der eingezeichneten Spiegelachse zu sehen ist, kehrt sich diese Figur um, so dass auf einen größeren Intervallsprung $H-D$ die ursprüngliche Pendelbewegung folgt (um ein Pärchen verkürzt), diesmal in umgekehrter Reihenfolge $G-D$ und $A-D$. Verkürzt man diese gesamte Figur auf vier Töne vor und vier Töne hinter der Spiegelachse, findet man sie mit identischen Bewegungsrichtungen wieder (Markierung 2), diesmal im Übergang der beiden Taktstriche. Da die letzte Sechzehntelnote $D$, mit der die Figur beginnt, aber noch dem ersten der beiden Takte zugeordnet wird, ist dieser Taktwechsel nur virtuell und nicht im Höreindruck erkennbar. Wie an Markierung 3 zu erkennen ist, schließt die gesamte Figur mit einer Wiederholung des Mittelsegments aus der ersten langen Notenkette (Markierung 1).

Es folgt eine neue Figur der Gitarre, bis sie in den Takten 9 bis 17 den drei anderen Instrumenten Gelegenheit gibt, mit Bruchstücken der Gitarrenlinie zu kommentieren. Ab Takt 18 steigt die Band endgültig ein und kehrt noch einmal zum Anfang zurück, wobei nun das Keyboard mit Unterstützung des Schlagzeugs die Stimmführung übernimmt, während die Gitarre Harmoniewechsel des Basses akkordisch untermalt. Hier erleben wir ein weiteres Prinzip von Dream Theaters Musik, nur sehr selten, als besonderer Effekt, Songteile wortwörtlich zu wiederholen. Im Regelfall wiederholter Refrains oder Strophen gewinnen sie lieber den jeweils zugrunde gelegten Ideen weitere Spielarten ab, um die Instrumentalparts damit abwechslungsreicher zu gestalten.

Der ab Takt 26 einsetzende B-Teil - nun auf der Quinte $A$ - komprimiert die bisherige Leitidee, den Grundton mit wechselnden Taktschwerpunkten zu umspielen, auf wenige Töne. Der Fokus liegt, nicht zuletzt dank des wieder aufgenommenen Unisono-Spiels der drei Melodieinstrumente, noch stärker auf den vom Schlagzeuggroove vorangetriebenen ungleichmäBigen Betonungen. Ausgangspunkt ist nun ein 9/16-Takt, der mal um ein, zwei, vier oder fünf Sechzehntel verlängert wird und auch bei mehrmaligem Hören eigenwillig zu zählen ist: 


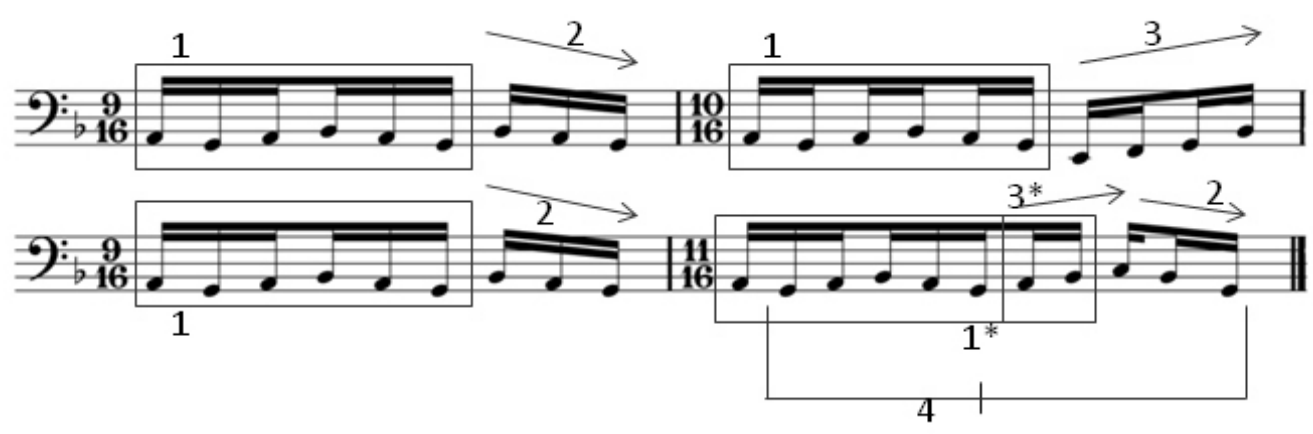

Abbildung 2: Dream Theater - »In The Presence Of Enemies«, Takt 26-29

Den Kern bildet eine Gruppe von sechs Tönen, die als wellenförmige Bewegung den Ausgangston A umspielt (siehe Ziffer 1). Darauf folgt eine nachgeschobene kürzere Einheit, zunächst mit drei abwärts geführten Noten (Ziffer 2), im zweiten Fall mit vier aufwärts strebenden Tönen (Ziffer 3). Der dritte Takt wiederholt wörtlich den ersten, bevor der vierte Takt auf engstem Raum die größte Variationsdichte entfaltet: Zum einen wird die mit einem Kasten markierte Sechstongruppe um zwei Töne verlängert (Ziffer $1^{*}$ ), die aufwärts steigen wie im zweiten Takt (Ziffer $3^{*}$ ), an die sich aber ausnahmsweise die bekannte Gruppe aus drei abwärts geführten Tönen anschließt (Ziffer 2). Vernachlässigt man im vierten Takt den ersten Ton (siehe Ziffer 4 mit Spiegelachse), lässt sich auch das bereits bekannte Prinzip einer figurativen Spiegelung von Motiven identifizieren.

Ein entscheidender Vorteil dieser Vorgehensweise ist die Steuerung der Wahrnehmung: Bei wachsender Komplexität eines Parameters - hier der rhythmischen Anordnung - sorgt ein anderes Element für stete Orientierung, in diesem Fall durch die Reduzierung des Tonumfangs sowie der Stimmführung im Unisono. Damit bleiben die Abweichungen vom bisherigen Schema erkennbar und das Gesamtbild wird nicht zu unübersichtlich. Hierbei ist zu berücksichtigen, dass aus einem mehr als fünfminütigen Vorspiel für einen 20 Minuten langen Song hier nur wenige Takte isoliert wurden, um ein Prinzip zu demonstrieren, das dennoch repräsentativ für ein Kompositionsprinzip von Dream Theater ist: die Strukturierung großer Zusammenhänge über melodische und rhythmische Motive. Im realen Kontext des Stückes ist diese Passage zwar deutlich komplexer, wird in rasantem Tempo ausgeführt und bezieht alle Instrumentenstimmen ein, die sich in unterschiedlichen Konstellationen verzahnen oder kommentieren. Dennoch ist - quasi auf der mikroskopischen Ebene - zu erkennen, wie sich mit diesem und weiteren Prinzipen große dramatische Zusammenhänge konzipieren lassen. Wenn man erstens bedenkt, dass hier nur der Kontext der instrumentalen Gestaltung berücksichtigt wurde, und zweitens die Möglichkeiten hinzu- 
nimmt, mit Songtexten musikalische Stimmungen zu ganzen Geschichten auszuformen, ahnt man das Potenzial einer Band wie Dream Theater.

\section{Komponieren für Virtuosen}

Häufig stehen junge Musiker zu Beginn ihrer Karriere vor der Schwierigkeit, nicht nur als originelle Songwriter auf sich aufmerksam zu machen, sondern auch unter Beweis stellen zu wollen, dass sie ihr Handwerk als Instrumentalisten verstehen. Dies kann einerseits zu Spannungen innerhalb einer Band führen, wenn z.B. ein Sologitarrist zum Missfallen der Kollegen bei Auftritten bevorzugt das Rampenlicht sucht. Andererseits können Songs zu überladen ausfallen, wenn sie zu vielen Zwecken auf einmal dienen sollen. Wie aber komponiert man, wenn man diese Phase schon lange hinter sich hat und - wie im Fall von Dream Theater - als Performer von Fachleuten und Fans dutzendfach mit Preisen ausgezeichnet wurde, so dass die Reputation respektabel nachgewiesen ist? Wie also gestaltet man Musik, bei der Virtuosität ein wesentlicher Bestandteil des Selbstverständnisses ist, mit der spieltechnisch aber nichts mehr bewiesen werden muss? Im Lauf der Jahre kristallisierten sich aus Dream Theaters Band-Repertoire bestimmte Songs zu festen Bestandteilen der Konzerte heraus, bei denen die Zuhörer Solopassagen in allen Einzelheiten genießen, so dass das Verlangen des Publikums nach gemeinsam zelebrierter Virtuosität einen festen Platz hat. Wie aber kalkulieren Musiker selbst ihre Stilmittel, wenn ihre Fähigkeit, außergewöhnlich schnell und kompliziert spielen zu können, nur eine unter vielen Möglichkeiten ist?

In diversen Gesprächen schilderte Jordan Rudess dem Autor, dass er selbst seine Wahrnehmung (und die seiner Kollegen) als Virtuosen durchaus ambivalent sieht. ${ }^{3}$ Einerseits beinhaltet diese Anerkennung ein Kompliment für solide Technik, die er fortlaufend trainieren muss, um sie zu erhalten. Trotz gegenteiliger Absichten können außergewöhnliche technische Fähigkeiten andererseits auch verängstigen und als Konkurrenzverhalten interpretiert werden. Die künstlerischen Konsequenzen dieser Ambivalenz sind musikalische und soziologische: Musikalisch macht Virtuosität den Versuch sichtbar, physische und psychische Einschränkungen zu überwinden, um musikalische Absichten möglichst direkt und pur auszudrücken. Ästhetischer Motor ist das übergeordnete Streben nach musikalischer Perfektion, das sich in diesem Fall in der kompositorischen Arbeit und ihrer performativen Inter-

3 Der Autor steht seit 2013 regelmäßig mit Jordan Rudess in Kontakt. 
pretation manifestiert. Daher umfasst die virtuose Beherrschung des Instruments gerade auch die leisen Töne, um kontrastierende, polychrome Stimmungen zu erzeugen. Die harmonische und rhythmische Komplexität, die gesteigerte Ansprüche an das musikalische Abstraktionsvermögen der Zuhörer stellt und damit bestimmte Publikumsteile besonders anspricht, wird damit zum Gegenpol melodischer und atmosphärischer Elemente, die sich emotional direkter vermitteln. Diese Balance aus Komplexität und Emotionalität entwickelte Dream Theater zum Markenkern ihrer Musik. Als erfahrene und etablierte Musiker kommt innen dabei ein Effekt zugute, den man »virtuelle« oder »imaginierte Virtuosität" nennen könnte. Im Wissen um ihr immer wieder bestätigtes Können lenkt beim Hören und Betrachten ihrer Arbeit diese virtuelle, imaginierte Virtuosität unsere Wahrnehmung: Wenn man sich bewusst macht, wie viel Musik sie bereits geschaffen und gespielt haben, kann ein neuer Song auf bestimmte Elemente konzentriert bleiben oder auch verzichten, wenn man ihn ins Verhältnis zu den Dutzenden bereits geschriebenen Songs setzt.

Dream Theaters eigenwillige Mischung gefühlvoller, balladesker Passagen mit schnellen, rhythmisch vertrackten Abschnitten machte sie zwar berühmt, doch wuchs mit ihrer Bekanntheit auch die Kritik an diesem Stil. Bezeichnenderweise bedienen sich kritische Fans und Rezensenten weniger musikalischer Argumente, sondern reduzieren ihre Einwände auf soziologische Stereotype: Während die komplexen Anteile als Kopfmusik für Nerds bezeichnet werden, gelten die emotionalen balladenartigen Songteile Gegnern dieser Musik als zu weich und weibisch. Darüber hinaus machte Rudess im Gespräch die aufschlussreiche Bemerkung, dass eine überschwängliche oder kritische Bewertung seiner Virtuosität auch genreabhängig ist und unterschiedliche soziale Orte hat: Während seine Fingerfertigkeit sowie seine Art der Klavierbegleitung im Kontext von Dream Theaters Progressive Metal bisweilen deutlich negative Reaktionen provoziert, erntet er mit exakt denselben Charakteristika höchstes Lob, zuletzt für seine Mitwirkung im Jazzrock-inspirierten Instumentaltrio mit Tony Levin und Marco Minneman.

Zieht man eine Zwischensumme dieser Beobachtungen, ermöglicht den Musikern von Dream Theater das Komponieren im Virtuosenkollektiv, auf eine Stilbreite zurückgreifen zu können, die ein Mitglied oder ein Album allein nicht leisten könnte. Im Umkehrschluss sind die musikalischen Gemeinsamkeiten aber auf den zuvor beschriebenen stilistischen Markenkern der Traditionen von Metal und Progressive Rock konzentriert. Andere Interessen der verschiedenen Bandmitglieder bleiben außen vor, wie die zahlreichen Nebenprojekte von Jordan Rudess, die Soloalben von Sänger James LaBrie und die Projektband The Jelly Jam von Bassist John Myung dokumentieren. 
Gemessen am übrigen Komplexitätsgrad von Rockmusik und Metal stellt sich abschließend die Frage, ob der Erfolg, den sich Dream Theater über Jahrzehnte bei einem Millionenpublikum weltweit erspielt haben, nicht im Widerspruch steht zu den erforderlichen Vorkenntnissen, diese Musik in allen Details zu verstehen, sowie zu den notwendigen technischen Fähigkeiten, ihre Songs nachzuspielen? Erstaunlicherweise besteht aber gerade kein Widerspruch zwischen den technischen Fertigkeiten der Musiker und ihrer Popularität. Stattdessen ist ihr Erfolg ein aussagekräftiges Phänomen unserer heutigen Musikkultur:

- Die allermeisten Vorgänge in unserer hoch technisierten Welt sind heute so kompliziert, dass sie sich dem Verständnis der meisten von uns entziehen. Wir vertrauen uns daher Experten an, die diese Komplexität transparent und rezipierbar aufbereiten, damit wir uns dazu verhalten können. Auf ihrem Gebiet komponieren Dream Theater einen so herausfordernden Grad an musikalischer Komplexität, dass es ihrer Interpretationsfähigkeit als Live-Musiker bedarf, uns die Handhabbarkeit, Sinnhaftigkeit und Genussfähigkeit solch komplizierter Strukturen - als Ausdruck von Kunst - vor Ohren und Augen zu führen.

- In einer Zeit scheinbar unbegrenzter digitaler Zugriffsmöglichkeiten haben wir uns inzwischen auf totale Manipulierbarkeit eingestellt, nicht nur, aber auch akustischer und visueller Artefakte. Ein entscheidender Faktor für den Erfolg von Dream Theater und anderen Virtuosen ist ihre Live-Präsenz, mit der uns die menschliche Gestaltung von Zusammenhängen bewiesen wird, deren Komplexität die meisten von uns überfordern würde.

- Bei aller Expertendistanz, die technische Fähigkeiten in der Praxis ausstrahlen können, schaffen gerade diese elaborierten Kunstmittel eine besondere Nähe zum Publikum. Eine solche Virtuosität wirkt nicht bedrohlich oder kalt, sondern ist ein entscheidendes Kommunikationsmittel um zu verdeutlichen, warum man genau solche Bands braucht und weshalb sie sich mit diesem Markenzeichen schon so lange im Geschäft halten können: Trotz aller technischen Mittel bleibt die menschliche Komponente die entscheidende Zutat von Musik. Im Fall von Dream Theater ist sie komplex und lebendig zugleich, was sie zu einem spannenden Rätsel macht. Einfache Lösungen werden schnell langweilig und gerade auch der Progressive Metal fordert seine Liebhaber mit hintergründigen Resultaten heraus. Für deren Gestaltung ist Virtuosität eine Schlüsselkompetenz. 


\title{
Literatur
}

Berg, Alban (1984). »Prospekt des >Vereins für musikalische Privataufführungen« In: Schönbergs Verein für musikalische Privataufführungen. Hg. v. Heinz-Klaus Metzger und Rainer Riehn (= Musik-Konzepte 36). München: edition text + kritik, S. 4-7.

Bork, Camilla (2010). »Virtuosität. «In: Lexikon Musik und Gender. Hg. v. Annette Kreutziger-Herr und Melanie Unseld. Kassel: Bärenreiter und München: Metzler, S. $510 f$.

Custodis, Michael (2016). Traditions, Inspirations, Contrasts. Jordan Rudess' »Explorations for Keyboard and Orchestra «, Online-Publikation Münster, http:// www.uni-muenster.de/imperia/md/content/musikwissenschaft/pdf/custodis_-_ jordan_rudess_explorations.pdf (Stand vom 20.4.2017).

Dahlhaus, Carl / Eggebrecht, Hans Heinrich (Hg.) (1967). Brockhaus Riemann Musiklexikon. Sachteil, Mainz: Schott.

Jütte, Daniel (2009). »Juden als Virtuosen. Eine Studie zur Sozialgeschichte der Musik sowie zur Wirkmächtigkeit einer Denkfigur des 19. Jahrhunderts. «In: Archiv für Musikwissenschaft 66, H. 2, S. 127-154.

Kuhnau, Johann (1700). Der musicalische Quack-Salber. Dresden: Riedel und Hoff.

Mattheson, Johann (1722). Critica Musica. Hamburg: Thomas von Wierings Erben.

Mozart, Leopold (1770). Versuch einer gründlichen Violinschule. Augsburg: Carl Stephenson (2. Aufl.).

Reimer, Erich (1972). »Virtuose. «In: Handwörterbuch der musikalischen Terminologie. Hg. von Hans Heinrich Eggebrecht. Freiburg i. Br.: Franz Steiner.

Riethmüller, Albrecht (2001): $\gg$ Die Verdächtigung des Virtuosen - Zwischen Midas von Akragas und Herbert von Karajan.« In: Virtuosen. Über die Eleganz der Meisterschaft. Hg. vom Herbert von Karajan-Center Wien. München: Paul Zsolnay, S. 100-124.

Stößel, Johann Christoph / Stößel, Johann David (1749). Kurzgefaßtes musicalisches Lexicon. Chemnitz: Stößel.

\begin{abstract}
While technical skills are often valued as a key feature for the artistic quality of rock music (especially in progressive rock and metal) virtuosity seems not the primary concern of musicians that are labeled virtuoso. Instead virtuosity is a receptional category of looking at musicians and their performance. To understand how musicians craft complex, technically ambitious pieces and why they balance the desire to create new challenges, the essay concentrates on the US-American band Dream Theater. Furthermore the paper describes how the band uses virtuosic passages as a tool for direct emotional interaction with their fans during live concerts.
\end{abstract}

